\title{
Die Zeitschrift für die gesamte Versicherungswissenschaft wird 100!
}

\author{
J.-Matthias Graf von der Schulenburg
}

Online publiziert: 5. Februar 2011

(C) Springer-Verlag 2011

Der wissenschaftliche Diskurs zwischen den vielfältigen Versicherungsdisziplinen hat eine sehr lange Tradition in Deutschland: 2011 veröffentlichen wir den 100. Band der Zeitschrift für die gesamte Versicherungswissenschaft (ZVersWiss). Zwar erblickte die ZVersWiss 1901, d.h. in dem Jahr, in dem in Deutschland das Versicherungsaufsichtsgesetz erlassen wurde, das erste mal das Licht der Welt, aber kriegsbedingt konnte die Zeitschrift in 10 Jahren nicht erscheinen. Mit dieser langen und verdienstvollen Historie gehört die ZVersWiss zu den ältesten versicherungswissenschaftlichen Zeitschriften der Welt. In diesen 100 Jahren bildet die Zeitschrift die Drehscheibe der versicherungswissenschaftlichen Diskussion in Deutschland: In ihr wurden die neuesten Ergebnisse der Forschung publiziert, kann der Diskus zwischen Wissenschaftlern untereinander und mit wissenschaftlich interessierten Praktikern nachgezeichnet werden und wurden die Regulierungen des Versicherungswesens aus juristischer, ökonomischer, mathematischer und naturwissenschaftlicher Sicht kommentiert. In den 100 Jahren haben der Zeitschrift viele Persönlichkeiten in dem Herausgebergremium gedient, allerdings kam die Zeitschrift in 110 Jahren mit 5 Schriftleitern aus. Über die ganze Zeit war die ZVersWiss eng mit dem Deutschen Verein für Versicherungswissenschaften e. V. verbunden. Im Laufe der Zeit hat die Zeitschrift ihre Form und den Verlag mehrfach geändert und ist inzwischen eine wissenschaftliche Zeitschrift mit einem „,doppelt-blinden Referee-Verfahren“ und einer Internetplattform der „online-first“-Publikation der Aufsätze.

Aber die Herausforderung einer langen Tradition ist nicht das Bewundern der Asche, sondern das Weitertragen der Glut. Entsprechend muss sich die Zeitschrift den neuen Trends in der Wissenschaft, den veränderten Bedürfnissen ihrer Leser und

J.-M. Graf von der Schulenburg ( $\varangle)$

Leibniz Universität Hannover, Königsworther Platz 1, 30167 Hannover, Deutschland

e-mail: jms@ivbl.uni-hannover.de 
den neuen Kommunikationstechniken öffnen. Mit dem Springer Verlag kam die Zeitschrift in den Genuss der Internetplattform des Verlages. Durch sie kann man Aufsätze der Zeitschrift runterladen. Dies geschieht pro Jahr etwa 5.000 Mal! Dabei ist ein kontinuierliches Ansteigen der Download-Häufigkeit zu verzeichnen. Die in der Zeitschrift publizierten Aufsätze erfahren eine Verbreitung wie nie zuvor.

Anspruch und ständige Herausforderung für die Zeitschrift ist auch die Förderung des Austausches zwischen der Wissenschaft und der Versicherungspraxis. Schon Henry Ford hat diese Symbiose treffend beschrieben: „Zusammenkunft ist ein Anfang. Zusammenhalt ist ein Fortschritt. Zusammenarbeit ist der Erfolg."

Der Erfolg der ZVersWiss war nur mit einem engagierten Herausgebergremium, fleißigen und profunden Autoren und tüchtigen Gutachtern möglich. Deshalb nutze ich dieses Jubiläumseditorial auch um den Autoren, aber insbesondere den Gutachtern zu danken. Als Gutachter haben sich zuletzt folgende Persönlichkeiten um die ZVersWiss verdient gemacht:

Stefan Barenberg, Bremen

Stephan Becher, Köln

Jens Bölscher, Braunschweig

David Bowles, Bielefeld

Christa Claes, Neu-Isenburg

Wolfgang Greiner, Bielefeld

Zdenko Grobenski, Bonn

Elmar Helten, München

Klaus-Dirk Henke, Berlin

Jan-Marc Hodek, Bonn

Stefan Homburg, Hannover

Susanne Klein, Hamburg

Torsten Körber, Göttingen

Simone Krummaker, Hannover

Bernd Lohse, Hannover

Ute Lohse, Hannover

Thomas Mittendorf, Hannover
Jürgen Petzold, Wilhelmshaven

Anne Prenzler, Hannover

Jens-Ole Rauh, Hannover

Sebastian Reddemann, Berlin

Andreas Richter, München

Hans-Gerd Ridder, Hannover

Wolfgang Michael Roth, Hannover

Heinrich Rudolf Schradin, Köln

Oliver Schöffski, Nürnberg

Helge Schumacher, Berlin

Christoph Schwarzbach, Berlin

Christian Thomann, Stockholm

Christoph Vauth, Bückeburg

Fred Wagner, Leipzig

Manfred Wandt, Frankfurt

Klaus-Peter Wiedmann, Hannover

Andy Zuchandke, Hannover

Nach 100 Bänden ZVersWiss wäre es eine reizvolle Aufgabe, einmal zu untersuchen, welchen Fortschritt die deutsche Versicherungswissenschaft gemacht hat und welche Themen die Autoren im Wandel der Zeit bewegt haben. „In der Wissenschaft gleichen wir alle nur den Kindern, die am Rande des Wissens hier und da einen Kiesel aufheben, während sich der weite Ozean des Unbekannten vor unseren Augen erstreckt" (Isaac Newton). Es gibt noch unendlich viel zu erforschen. Das Versicherungswesen bleibt dabei ein interessantes Untersuchungsobjekt, da es so unterschiedliche wissenschaftliche Disziplinen betrifft, und ein Grundbedürfnis der Menschen zum Gegenstand hat: Sicherheit. 